Ergod. Th. \& Dynam. Sys. (1981), 1, 389-412

Printed in Great Britain

\title{
Maximally highly proximal generators of minimal flows
}

\author{
JOSEPH AUSLANDER AND JAAP VAN DER WOUDE \\ Department of Mathematics, University of Maryland, College Park, MD 20742, \\ USA; and Stichting Mathematisch Centrum, Amsterdam, The Netherlands
}

(Received July 1981 and revised 2 October 1981)

\begin{abstract}
We study minimal flows and their extensions by means of the associated maximally highly proximal flows. These, in turn, can be represented by highly proximal generators, which are certain subsets of the universal minimal flow. From this point of view we obtain information on relative disjointness, coalescence, the Bronstein property, and RIC extensions.
\end{abstract}

\section{Introduction}

In this paper we study a class of minimal flows which can be defined in terms of certain subsets of the universal minimal flow. In [3], the highly proximal extensions were introduced. These can be described as the extensions for which a fibre is shrunk uniformly to a point. To every minimal flow there is associated a unique 'maximally highly proximal' (MHP) minimal flow which, in turn, is defined by a 'maximally highly proximal generator', a subset of the universal minimal flow $M$, whose orbit closure in the 'hyperspace' $2^{M}$ is the given flow.

The present paper is devoted to an intensive study of these flows and some related questions. In $\S 1$, we show that $2^{M}$ admits an associative multiplication. In addition to its application here, this semigroup structure may also be useful in other problems in topological dynamics. In $\$ 2$, the main objects of interest are defined and studied. We review relevant concepts and results from [3], and give several alternative characterizations of maximally highly proximal flows and generators. It is shown (when the acting group has the discrete topology) that there are no non-trivial distal maximally highly proximal flows, and coalescence of MHP flows is discussed. Since disjointness is a 'highly proximal invariant', it is natural to consider disjointness and relative disjointness from the point of view of MHP generators, and this is done in $\S 3$. We also consider some questions concerning RIC extensions and the Bronstein property, and use the results obtained to discuss disjointness of minimal flows more general than the MHP flows ( $\$ 4)$.

We now establish some terminology and notations. A flow is a continuous action of a topological group $T$ on a compact Hausdorff space $X$. We write $(X, T)$, or just $X$, for a flow and denote the action of $t \in T$ on $x \in X$ by

$$
(x, t) \mapsto x t .
$$


The flow $X$ is minimal if the orbit closure $\overline{x T}=X$ for all $x \in X$. The trivial (one-point) flow will be denoted by 1 . If $X$ and $Y$ are flows (with the same acting group $T$ ), a homomorphism from $X$ to $Y$ is a continuous equivariant map $\phi: X \rightarrow Y$ :

$$
\phi(x t)=\phi(x) t, \quad \text { for } x \in X, t \in T \text {. }
$$

The term 'extension' is a synonym for homomorphism.

If $(X, T)$ is a (not necessarily minimal) flow, its enveloping semigroup, $E$ or $E(X)$, is the closure of the $T$ in $X^{X}$ (the collection of all maps from $X$ to itself, provided with the topology of pointwise convergence). $E$ is a semigroup of (not, in general, continuous) maps of $\boldsymbol{X}$ onto $\boldsymbol{X}$.

For a fixed group $T$, there is an isomorphically unique universal minimal flow $M$. Its defining property is that every minimal flow $(X, T)$ is a homomorphic map ('factor') of $M$. The flow $M$ is isomorphic with a minimal right ideal in $E(M)$. Thus $M$ is itself endowed with a semigroup structure, and acts on any minimal flow. We write $J$ for the set of idempotents in $M$ and choose $u \in J$. (This idempotent $u$ will be fixed in this paper.) If $G=M u$ then $G$ is a group (which can be identified with the group of automorphisms of $(M, T)$ ), and every $p \in M$ can be uniquely decomposed as $p=\alpha v$, when $\alpha \in G$ and $v \in J$. See [5] for further details and as a general reference.

1. $2^{M}$ as a semigroup

If $(X, T)$ is a flow, then $T$ naturally acts on the 'hyperspace' $2^{X}$, the space of non-empty closed subsets of $X$, provided with the Hausdorff topology. A quasifactor of $X$ is a minimal subset of the flow $\left(2^{X}, T\right)$. If $X$ is minimal, the universal minimal flow $M$ acts on $2^{X}$ by means of the 'circle operation': if $A \in 2^{X}$ and $p \in M$, then $A \circ p \in 2^{X}$ is defined by

$$
A \circ p=\lim A t_{i}
$$

where $\left(t_{i}\right)$ is a net in $T$ with $t_{i} \rightarrow p$ (see [7] and [3]).

In particular, $M$ acts on $2^{M}$ via the circle operation. We now show that the circle operation can be extended so that $2^{M}$ (and, in fact, all subsets of $M$ ) becomes a semigroup.

If $C \in 2^{M}$ and $\varnothing \neq D \subset M$, we define

$$
C \circ D=\bigcup[C \circ d \mid d \in D] .
$$

(Note that if $d \in M, C \circ\{d\}=C \circ d \supset C d$.)

(1.1) Lemma. (1) If $C, D \in 2^{M}$, then $C \circ D \in 2^{M}$ ('the product of two closed sets is closed').

(2) If $C \in 2^{M}, D \subset M$, then $C \circ \bar{D}=\overline{C \circ D}$.

Proof. The straightforward proof of (1) is omitted. If $C$ and $D$ are as in (2), then

$$
C \circ D \subset C \circ \bar{D}
$$

which is closed by (1), so

$$
\overline{C \circ D} \subset C \circ \bar{D}
$$


Let $q \in C \circ \bar{D}$, so $q \in C \circ \delta$, for $\delta \in \bar{D}$. Let $\left(d_{n}\right) \subset D$ be a net with $d_{n} \rightarrow \delta$. Then there are $q_{n} \in C \circ d_{n}$ with $q_{n} \rightarrow q$. Thus

$$
q_{n} \in C \circ d_{n} \subset C \circ D \text { and } q \in \overline{C \circ D}
$$

(1.2) LEMMA. If $C, D \in 2^{M}$ and $E \subset M$, then

$$
C \circ(D \circ E)=(C \circ D) \circ E \text {. }
$$

Proof. Let $f \in D \circ E$. Then $f \in D \circ \varepsilon$, for some $\varepsilon \in E$. We show

$$
C \circ f \subset(C \circ D) \circ \varepsilon
$$

Let $\left(d_{n}\right) \subset D,\left(t_{n}\right) \subset T$ be nets with

$$
t_{n} \rightarrow \varepsilon, \quad d_{n} t_{n} \rightarrow f
$$

Then

$$
C \circ d_{n} t_{n} \rightarrow C \circ f
$$

and

$$
C \circ d_{n} t_{n} \subset(C \circ D) t_{n} \rightarrow(C \circ D) \circ \varepsilon .
$$

Hence

$$
C \circ f \subset(C \circ D) \circ \varepsilon
$$

and therefore

$$
C \circ(D \circ E) \subset(C \circ D) \circ E \text {. }
$$

To prove the opposite inclusion, let $\varepsilon \in E$, and let $\left(t_{n}\right)$ be a net in $T$ with $t_{n} \rightarrow \varepsilon$. Then

$$
(C \circ D) t_{n} \rightarrow(C \circ D) \circ \varepsilon
$$

and also

$$
(C \circ D) t_{n}=C \circ\left(D t_{n}\right) \rightarrow C \circ(D \circ \varepsilon)
$$

so

$$
(C \circ D) \circ \varepsilon \subset C \circ(D \circ E)
$$

and hence

$$
(C \circ D) \circ E \subset C \circ(D \circ E) \text {. }
$$

(1.3) THEOREM. With respect to the circle operation, $2^{M}$ is a semigroup in which multiplication is continuous in the second argument.

Proof. The continuity assertion (if $D_{n} \rightarrow D$ then $C \circ D_{n} \rightarrow C \circ D$ ) is straightforward. The other properties follow from (1.1) and (1.2).

Now, we extend the circle operation to all non-empty subsets of $M$ by defining

$$
C \circ D=\bar{C} \circ D \quad(C, D \subset M)
$$

If $C, D, E \subset M$, then

$$
(C \circ D) \circ E=(\overline{\bar{C} \circ D}) \circ E=(\bar{C} \circ \bar{D}) \circ E=\bar{C} \circ(\bar{D} \circ E)=\bar{C} \circ(D \circ E)=C \circ(D \circ E),
$$

so multiplication is still associative. 


\section{Maximally highly proximal flows and generators}

We begin this section by reviewing the notion of highly proxinal extensions. If $\phi: X \rightarrow Y$ is a homomorphism, then $2^{\phi}$ is the closed invariant subset of $2^{X}$ defined by

$$
2^{\phi}=\left[A \in 2^{X} \mid A \subset \phi^{-1}(y), \text { for some } y \in Y\right] .
$$

Write $\bar{\phi}$ for the homomorphism of $2^{\phi}$ to $Y$ defined by $\bar{\phi}(A)=y$, where $A \subset \phi^{-1}(y)$.

Recall that $x, x^{\prime} \in X$ are proximal if there is a $z \in X$ and a net $\left(t_{i}\right) \subset T$ with

$$
x t_{i} \rightarrow z \text { and } \quad x^{\prime} t_{i} \rightarrow z \text {. }
$$

The homomorphism $\phi: X \rightarrow Y$ is called proximal if $x$ and $x^{\prime}$ are proximal whenever $\phi(x)=\phi\left(x^{\prime}\right)$.

Let $\phi: X \rightarrow Y$ be a homomorphism of minimal flows. Then $\phi$ is called highly proximal (HP) if it satisfies one of the following equivalent conditions:

(a) $\phi$ is irreducible (if $A \subset X$ such that $\phi(A)=Y$, then $\bar{A}=X$ ).

(b) The homomorphism $\bar{\phi}: 2^{\phi} \rightarrow Y$ is proximal.

(c) $2^{\phi}$ has a unique minimal subset.

(d) There is a $y \in Y$ and a net $\left(t_{i}\right)$ in $T$ such that $\left(\phi^{-1}(y) t_{i}\right)$ converges (in $\left.2^{X}\right)$ to a singleton.

(e) If $y \in Y, p \in M$ and $x \in \phi^{-1}(y)$, then $\phi^{-1}(y) \circ p=\{x p\}$.

(The equivalence of $(a),(d)$ and $(e)$ is shown in [3]. It is trivial that $(e) \Rightarrow(b) \Rightarrow(c)$, and $(c) \Rightarrow(d)$ follows from the fact that $X$ is a minimal subflow of $2^{\phi}$.)

It is an immediate consequence of $(b)$ that highly proximal extensions are proximal. The converse fails (see [3]). A minimal flow $X$ is said to be maximally highly proximal (MHP) if it admits no non-trivial highly proximal extensions. (That is, if $\phi: Z \rightarrow X$ is highly proximal, then $\phi$ is an isomorphism.) In [3], it is shown that to very minimal flow $X$ there is associated a unique MHP flow $X^{*}$ which is an HP extension of $X$. It follows that the class of minimal flows (with acting group $T$ ) is partitioned with 'HP equivalence classes' - two flows are HP equivalent if their associated MHP flows are isomorphic.

The MHP flow $X^{*}$ is obtained as follows: if $\gamma: M \rightarrow X$ is a homomorphism and $x \in X$, then

$$
X^{*}=\left[\gamma^{-1}(x) \circ p \mid p \in M\right] \text {. }
$$

It follows from this representation that to every homomorphism of minimal flows there is induced a homomorphism of their maximally highly proximal extensions. In fact, if $\phi: X \rightarrow Y, \gamma: M \rightarrow X, \delta=\phi \gamma: M \rightarrow Y, x \in X$, and $y=\phi(x)$, then $\phi^{*}: X^{*} \rightarrow Y^{*}$ is defined by

$$
\phi^{*}\left(\gamma^{-1}(x) \circ p\right)=\delta^{-1}(y) \circ p \quad(p \in M) .
$$

Moreover, the map $\gamma^{-1}(x) \circ p \rightarrow x p$ of $X^{*}$ to $X$ is HP. It is also shown in [3] that a minimal flow $X$ is MHP if and only if some (equivalently every) homomorphism from $M$ to $X$ is open. (It follows that every homomorphism from a minimal flow to $X$ is open. In particular, $\phi^{*}: X^{*} \rightarrow Y^{*}$ is open.)

In case $T$ is endowed with the discrete topology we shall characterize the MHP minimal flows in terms of the topology of the phase space. Recall that a topological space is extremely disconnected if the closure of every open set is open. 
(2.1) Theorem. Let $T$ be a discrete group. A minimal flow $(X, T)$ is MHP if and only if $X$ is extremely disconnected.

Proof. If $Z$ is any compact Hausdorff space there is an associated 'Gleason space' $Z_{\Sigma}$ which is compact Hausdorff and extremely disconnected, and there is a surjective continuous map from $Z_{\Sigma}$ to $Z$ [1]. Moreover, if $T$ is a discrete group which acts minimally on $Z$, there is a minimal action of $T$ on $Z_{\Sigma}$, and the $\operatorname{map} Z_{\Sigma} \rightarrow Z$ is a homomorphism. Therefore the universal minimal flow $M$ for the discrete group $T$ is extremely disconnected. Now if $(X, T)$ is MHP, then the homomorphism $\gamma: M \rightarrow X$ is open, and it follows easily that $X$ is extremely disconnected. Conversely, if $(X, T)$ is a minimal flow with $X$ extremely disconnected, then it is an elementary exercise $[1 i, 17 P]$ that there is no irreducible continuous map with codomain $X$. Thus $(X, T)$ is MHP, and the proof is completed.

Note that, if $X$ is a minimal flow and, as above, $X_{\Sigma}$ is the minimal flow on the Gleason space, then (still assuming the acting group $T$ is discrete) $X_{\Sigma}$ is isomorphic with $X^{*}$. For the homomorphism from $\boldsymbol{X}_{\Sigma}$ to $\boldsymbol{X}$ is irreducible, hence HP, and $\boldsymbol{X}_{\Sigma}$ is MHP by (2.1), so $X_{\Sigma} \cong X^{*}$.

(2.2) Theorem. Let $T$ be a discrete group. If $(X, T)$ is distal and MHP, then $X$ is a finite space (and so $(X, T)$ is equicontinuous).

Proof. Let $\left(X_{\text {eq }}, T\right)$ denote the maximal equicontinuous factor of $(X, T)$. Now any homomorphism whose domain is a distal minimal flow is open, and it follows that $\boldsymbol{X}_{\mathrm{eq}}$ is extremely disconnected. An equicontinuous flow has a homogeneous phase space [5] and any extremely disconnected homogeneous compact space must be finite [4]. So $X_{\text {eq }}$ is finite.

If $X$ is infinite, the canonical homomorphism $\pi: X \rightarrow X_{\mathrm{eq}}$ is non-trivial and, by the Furstenberg structure theorem [6], there is a factor $Y^{\prime}$ of $X$ which is a non-trivial almost periodic extension of $X_{\text {eq }}$. But, as is easily checked, an almost periodic extension of a flow on a finite space is equicontinuous. (See [8] for a more general result.) That is, $\boldsymbol{X}^{\prime}$ is an equicontinuous flow. This contradicts the definition of $\boldsymbol{X}_{\text {eq }}$ as the maximal equicontinuous factor of $X$.

A minimal flow is coalescent if every endomorphism is an automorphism. We now find conditions for the MHP extension of a distal minimal flow to be coalescent. First, we require a lemma.

(2.3) Lemma. Let $X$ and $X^{\prime}$ be minimal flows with $X$ distal, and let $\pi: X^{\prime} \rightarrow X$ be a proximal extension.

(1) If $\phi^{\prime}: X^{\prime} \rightarrow X^{\prime}$ is an endomorphism, then there is an endomorphism $\phi$ of $X$ such that $\pi \phi^{\prime}=\phi \pi$.

(2) Let $\phi$ and $\phi^{\prime}$ be endomorphisms of $X$ and $X^{\prime}$ respectively with $\pi \phi^{\prime}=\phi \pi$. Suppose $\phi$ is not an automorphism. Then $\phi^{\prime}$ is not an automorphism.

Proof. (1) If $x \in X$, define

$$
\phi(x)=\pi \phi^{\prime}\left(x^{\prime}\right)
$$

where $x^{\prime} \in \pi^{-1}(x)$. To see that $\phi$ is well defined let $y^{\prime} \in \pi^{-1}(x)$. Then $x^{\prime}$ and $y^{\prime}$ are 
proximal, so $\pi \phi^{\prime}\left(x^{\prime}\right)$ and $\pi \phi^{\prime}\left(y^{\prime}\right)$ are proximal. Since $X$ is distal,

$$
\pi \phi^{\prime}\left(x^{\prime}\right)=\pi \phi^{\prime}\left(y^{\prime}\right)
$$

(2) Let $x_{1}, x_{2} \in X$, with $x_{1} \neq x_{2}$ and $\phi\left(x_{1}\right)=\phi\left(x_{2}\right)$. Let $x_{1}^{\prime}, x_{2}^{\prime} \in X^{\prime}$ such that

$$
\pi\left(x_{1}^{\prime}\right)=x_{1}, \quad \pi\left(x_{2}^{\prime}\right)=x_{2},
$$

and $\left(x_{1}^{\prime}, x_{2}^{\prime}\right)$ is an almost periodic point of $X \times X$. Now, if $\phi^{\prime}\left(x_{1}^{\prime}\right) \neq \phi^{\prime}\left(x_{2}^{\prime}\right)$, then $\left(\phi^{\prime}\left(x_{2}^{\prime}\right), \phi^{\prime}\left(x_{2}^{\prime}\right)\right)$ is an almost periodic point of $X \times X$ with

$$
\pi \phi^{\prime}\left(x_{1}^{\prime}\right)=\phi \pi\left(x_{1}^{\prime}\right)=\phi\left(x_{1}\right)=\phi\left(x_{2}\right)=\phi \pi\left(x_{2}^{\prime}\right)=\pi \phi^{\prime}\left(x_{2}^{\prime}\right) .
$$

This contradicts the assumption that $\pi$ is proximal.

(2.4) TheOREM. Let $X$ be a distal minimal flow. Then $X^{*}$ is coalescent if and only if $X$ is coalescent.

Proof. Suppose $X$ is not coalescent. Let $\phi$ be an endomorphism of $X$ which is not an automorphism, and let $\phi^{*}$ be the induced endomorphism of $X^{*}$. By (2) of (2.3), $\phi^{*}$ is not an automorphism.

Suppose $X$ is coalescent, and let $\phi^{*}$ be an endomorphism of $X^{*}$. By lemma 2.3 (1), $\phi^{*}$ projects to an endomorphism $\phi$ of $X$ which, by assumption, must be an automorphism.

Let $\psi^{*}$ be the lift to $X^{*}$ of $\phi^{-1}$. Then, if $\pi: X^{*} \rightarrow X$ is the natural homomorphism,

$$
\pi \psi^{*} \phi^{*}=\pi
$$

and, if $x^{*} \in X^{*}, \psi^{*} \phi^{*}\left(x^{*}\right)$ is proximal with $x^{*}$ (since $\pi$ is HP, hence proximal). But this is impossible unless $\psi^{*} \phi^{*}$ is the identity.

Since there are non-coalescent distal minimal flows [9], theorem 2.4 shows that there are MHP minimal flows which are not coalescent.

As we have noted, an MHP minimal flow can be 'represented' as a quasi-factor of the universal minimal flow $M$. This motivates the following definition. Fix an idempotent $u$ in $M$. A non-empty subset $C$ of $M$ will be called a maximal highly proximal generator ('MHP generator') if $u \in C$ and $C \circ p=C$ for all $p \in C$ (so that, if $p \in M, C \circ p=C$ if and only if $p \in C$ ).

If $C$ is a subset of $M$, with $u \in C$ and $C \circ u=C$, then the following conditions are equivalent [3, theorem 1.3$]$ :

(i) $C$ is an MHP generator;

(ii) the sets $[C \circ p \mid p \in M]$ form a partition of $M$;

(iii) $C \circ p \subset C$ for all $p \in C$.

Thus, if $X$ is a minimal flow $x \in X$, and $\gamma: M \rightarrow X$ is defined by

$$
\gamma(p)=x p
$$

then

$$
C=\gamma^{-1}(x) \circ u
$$

satisfies (ii) and so is an MHP generator. Also, if $C$ is an MHP generator, the homomorphism $p \mapsto C \circ p$ is open (by condition (ii) above). Hence the minimal flow $\overline{C T}$ is in fact an MHP flow. 
Note that if $\varnothing \neq D \in 2^{M}$ is an idempotent $(D \circ D=D)$ and $C=D \circ u$, then

$$
C \circ C=D \circ u \circ D \circ u=D \circ D \circ u=D \circ u=C .
$$

Since $D$ is closed and $D D \subset D, D$ contains an idempotent $v$, so $u=v u \in C$, and therefore $C$ is an MHP generator.

The next theorem correlates algebraic properties of MHP generators with the dynamical properties of the minimal flows they define.

(2.5) TheOREM. Let $C$ and $D$ be MHP generators and let $\mathscr{C}=\overline{C T}, \mathscr{D}=\overline{D T}$. Then

(1) If $\mathrm{g} \in \mathrm{G}, \mathrm{gC} \circ \mathrm{g}^{-1}$ is an MHP generator.

(2) There is a homomorphism $\phi: \mathscr{C} \rightarrow \mathscr{D}$ with $\phi(C)=D$ if and only if $C \subset D$.

(3) The flows $\mathscr{C}$ and $\mathscr{D}$ are isomorphic if and only if $\beta \mathscr{C}=\mathscr{D}$, for some $\beta \in G$.

(4) The flow $\mathscr{C}$ is regular if and only if $g C \in \mathscr{C}$, for all $\mathrm{g} \in G$ if and only if $\mathrm{g}^{-1} \mathrm{C} \circ \mathrm{g}=\mathrm{C}$, for all $\mathrm{g} \in \mathrm{G}$.

(Recall that a minimal flow $X$ is regular [2] if, whenever $x, x^{\prime} \in X$ with $\left(x, x^{\prime}\right)$ an almost periodic point of $X \times X$, then there is an automorphism of $X$ taking $x$ to $x^{\prime}$.) Proof. (1) Let $E=g C \circ g^{-1}$. Then $u \in E$, and

$$
E \circ u=g C \circ g^{-1} \circ u=g C \circ g^{-1}=E .
$$

Also

$$
E \circ E=\left(g C \circ g^{-1}\right) \circ\left(g C \circ g^{-1}\right)=g C \circ u C \circ g^{-1}=g C \circ C \circ g^{-1}=g C \circ g^{-1}=E .
$$

(2) If $C \subset D$ and $p \in M$ such that $C \circ p=C$, then $p \in C \subset D$, so

$$
D \circ p=D \text {. }
$$

If $p, q \in M$ with $C \circ p=C \circ q$, then

$$
D \circ p \cap D \circ q \neq \varnothing,
$$

so

$$
D \circ p=D \circ q
$$

Then

$$
\phi(C \circ p)=D \circ p \quad(p \in M)
$$

defines a homomorphism from $\mathscr{C}$ to $\mathscr{D}$ with $\phi(C)=D$. Conversely, if $\phi$ is such a homomorphism and $p \in C$, then

$$
C \circ p=C,
$$

so

$$
D \circ p=\phi(C) \circ p=\phi(C \circ p)=\phi(C)=D
$$

and $p \in D$.

(3) Sufficiency is clear. Suppose $\phi: \mathscr{C} \rightarrow \mathscr{D}$ is an isomorphism. Then

$$
\phi(C)=D \circ \beta,
$$

for some $\beta \in G$. Let

$$
D^{\prime}=\beta^{-1} D \circ \beta
$$


By (1) $D^{\prime}$ is an MHP generator and, if $\mathscr{D}^{\prime}=\overline{D^{\prime} T}, \beta^{-1} \phi: \mathscr{C} \rightarrow \mathscr{D}^{\prime}$ is an isomorphism with

$$
\beta^{-1} \phi(C)=D^{\prime}
$$

By (2) $C=D^{\prime}$ so

$$
\beta^{-1} \phi(C)=C
$$

and

$$
\phi(C)=D \circ \beta=\beta D^{\prime}=\beta C .
$$

If follows that $\beta \mathscr{C}=\mathscr{D}$.

(4) For $g \in G, g C \in \mathscr{C}$ if and only if $g C \circ g^{-1} \in \mathscr{C}$ if and only if $g C \circ g^{-1}=C$ (since $u \in C \cap g C \circ g^{-1}$ ). Suppose $\mathscr{C}$ is regular. Then, if $g \in G$, there is an automorphism $\phi$ of $\mathscr{C}$ such that

$$
\phi(C)=C \circ g^{-1} .
$$

Then $C \circ p \rightarrow g \phi(C \circ p)(p \in M)$ defines an isomorphism of $\mathscr{C}$ onto the flow $g \mathscr{C}$. Since $\mathrm{gC} \circ \mathrm{g}^{-1}$ is an MHP generator whose orbit closure $\mathrm{g} \mathscr{C}$ is isomorphic with $\mathscr{C}$ we have (by (2))

$$
g C \circ g^{-1}=C .
$$

Conversely, if $g C \circ g^{-1}=C$ for $g \in G$, then $g C=C \circ g \in \mathscr{C}$ so left multiplication by $g$ defines an automorphism of $\mathscr{C}$ taking $C$ to $C \circ g$. Hence $\mathscr{C}$ is regular.

If $C$ is an MHP generator with $\mathscr{C}=\overline{C T}$, and $\phi: \mathscr{C} \rightarrow \mathscr{C}$ is an automorphism, then $\phi(C)=g C$ for some $g \in G$ (by (3) of (2.5)). If $h \in G$, then clearly $\phi(C)=h C$ if and only if $h=g \alpha$ for some $\alpha \in A=C \cap G$. Thus the group of automorphisms of $\mathscr{C}$ may be identified with the set

$$
\left[g A \in G / A \mid g^{-1} C \circ g=C\right] \text {. }
$$

We now consider the structure of MHP generators as well as the (apparently more difficult) question of when an almost periodic point of $2^{M}$ is an MHP generator.

The ' $\tau$-topology' on the group $G=M u$ is defined by the closure operator

$$
\mathrm{cl}_{\tau} A=A \circ u \cap G=(A \circ u) u,
$$

for $A \subset G$. $G$ provided with the $\tau$-topology is a compact $T_{1}$ space. Group multiplication is (separately) continuous, as is inversion.

(2.6) Lemma. (1) Let $C$ be in $M$ with $C \circ u=C$. Then $C u=C \cap G$ is $\tau$-closed in $G$.

(2) Let $H, L \subset M$. Then

$$
(H \circ L \circ u) u=(H \circ u) u(L \circ u) u .
$$

Proof. (1) $C u \subset(C u \circ u) u \subset(C \circ u \circ u) u \subset C \circ u \cap G=C \cap G=C u$, so

$$
C u \circ u \cap G=(C u \circ u) u=C u
$$

is $\tau$-closed.

(2) We may suppose that $H \circ u=H$ and $L \circ u=L$ and show that

$$
(H \circ L) u=H u L u \text {. }
$$


Now

$$
H u L u \subset H \circ u \circ L \circ u=H \circ L \circ u=H \circ L
$$

and, since $H u L u \subset G$, we have

$$
H u L u \subset(H \circ L) u .
$$

Let $\alpha \in(H \circ L) u$, so $\alpha=p u$, with $p \in H \circ L$. Then $p \in H \circ l$, for $l \in L$, and

$$
\alpha=p u \in(H \circ l) \circ u=H \circ l u,
$$

with $l u \in L u$. Hence

$$
\alpha(l u)^{-1} \in H \cap G=H u,
$$

so

$$
\alpha \in(H u) l u \subset(H u)(L u) .
$$

(2.7) THEOREM. Let $C$ be an MHP generator. Then

(1) $A=C \cap G=C u$ is a $\tau$-closed subgroup of $G$.

(2) If $K=C \cap J$, then $C=A K=A \circ K$.

Proof. (1) $A A \subset(C \circ u \circ C \circ u) u=C u=A$, so $A$ is a $\tau$-closed subsemigroup of $G$, and so $[5,2.11] A$ is a group.

(2) Let $p \in C$ and $v \in J$ with $p v=p$. Then

$$
C=C \circ p=C \circ p v=C \circ p \circ v=C \circ v,
$$

so $v=u v \in C$. Thus $v \in C \cap J=K$, and $p=p u v \in A K$. Therefore

$$
C \subset A K \subset A \circ K=C \circ C=C \text {. }
$$

For the remainder of this section, we are concerned with the converse direction.

(2.8) Lemma. Let $A$ be a subgroup of $G$ and let $K$ be a non-empty subset of $J$. Suppose that $C=A K$ satisfies $C \circ u=C$. Then $u \in K, C \cap G=A, C \cap J=K$, and $A$ is $\tau$-closed.

Proof. Let $v \in K$. Then

$$
v=u v \in A K=C,
$$

and

$$
u=v u \in C \circ u=C .
$$

Since every $p \in M$ has a unique decomposition

$$
p=\alpha w \quad(\alpha \in G, w \in J),
$$

it follows that $u \in K$ and also that

$$
C \cap G=A, \quad C \cap J=K .
$$

Finally

$$
A \circ u \cap G \subset C \circ u \cap G=C \cap G=A=A u \subset A \circ u \cap G,
$$

so $A$ is $\tau$-closed.

If $C=A K$ is as in (2.8) it follows from (2.6) (2) that the 'group part' of any power of $C$ (in the semigroup $\left(2^{M}, \circ\right)$ ) is $A$. 
(2.9) Theorem. Let $A$ be a $\tau$-closed subgroup of $G$, let $\varnothing \neq K \subset J$ and let $C=$ $A \circ K \circ u$. Suppose that either

(1) $K \circ A \circ K \circ u \subset G K$ and $(K \circ u) u \subset A$, or

(2) $K \circ A \circ u \cup K \circ K \circ u \subset C$.

Then $C$ is an MHP generator.

Proof. (1) From (2.6),

$$
C u=(A \circ K \circ u) u=(A \circ u) u(K \circ u) u \subset A A=A .
$$

Similarly,

$$
(K \circ A \circ K \circ u) u \subset A,
$$

so (since $K \circ A \circ K \circ u \subset G K$ ),

Then

$$
K \circ A \circ K \circ u \subset A K .
$$

$$
\begin{aligned}
C \circ C & =C \circ C \circ u=(A \circ K \circ A \circ K \circ u) \circ u \subset(A \circ A K) \circ u \subset(A \circ A \circ K) \circ u \\
& =(A \circ u \circ K) \circ u=A \circ K \circ u=C .
\end{aligned}
$$

Note that, if $v \in K$,

$$
u=v u v u \in K \circ A \circ K \circ u=G K,
$$

so $u \in K$. Clearly, $C \circ u=C$ and $C$ is an MHP generator.

(2) If $v \in K$,

$$
u=u v u \in A \circ K \circ u=C \text { and } C \circ u=C .
$$

Also

$$
\begin{aligned}
C \circ C & =A \circ K \circ A \circ K \circ u=A \circ K \circ A \circ u \circ K \circ u \subset A \circ A \circ K \circ u \circ K \circ u \\
& =A \circ A \circ K \circ K \circ u \subset A \circ A \circ A \circ K \circ u=A \circ u \circ K \circ u=A \circ K \circ u=C .
\end{aligned}
$$

Note that if $C$ satisfies the hypotheses of (i), then $C=A L$, where $L=C \cap J \subset K$. If $K \circ u \cap J=K$, then $C \cap J=K$ and $C=A K$.

(2.10) COROLlary. If $A$ is a $\tau$-closed subgroup of $G$ such that $(J \circ u) u \subset A$, then $C=A \circ J \circ u$ is an MHP generator.

(2.11) Corollary. Let $A$ be a $\tau$-closed subgroup of $G$, let $K \subset J$ and let $C=A K$. Suppose $C \circ u=C$, and that $K \circ A \circ K \circ u \subset G K$. Then

$$
C=A K=A K \circ u=A \circ K \circ u=A \circ K
$$

is an MHP generator.

Proof. $(K \circ u) u \subset(C \circ u) u=C u=A$, so $A \circ K \circ u$ is an MHP generator. But, as in the proof of $(2)$ in (2.9),

$$
K \circ A \circ K \circ u \subset A K=C,
$$

so

$$
A \circ K \circ u \subset K \circ A \circ K \circ u \subset C=C \circ u \subset A \circ K \circ u,
$$

and

$$
A \circ K \circ u=C .
$$


(2.12) THEOREM. Let $C=A K$, where $A$ is a $\tau$-closed subgroup of $G$ and $\varnothing \neq K \subset J$. Suppose $C \circ u=C$. Then the following are equivalent:

(1) $C$ is a MHP generator;

(2) $C \circ C \cap J \subset K$;

(3) $A \circ K \cup K \circ A \cup K \circ K \subset C$.

Proof. It is clear that (1) implies (2) and (3). Suppose (2) holds. Let $p \in C \circ C, p=\beta v$, $\beta \in G, v \in J$. Since $(C \circ C) u \subset A(2.6(2)), \beta \in A$ and

$$
v \in \beta^{-1} C \circ C \cap J=C \circ C \cap J \subset K \text {. }
$$

Thus

$$
C \circ C \subset(C \circ C \cap G)(C \circ C \cap J)=A K=C,
$$

and (1) is proved. If (3) holds, then

$$
\begin{aligned}
C \circ C= & A K \circ A K \subset A \circ(K \circ A) \circ K \subset A \circ(A K) \circ K \subset A \circ A \circ(K \circ K) \\
& \subset A \circ A \circ A \circ K \subset A \circ u \circ K=A \circ K \subset C .
\end{aligned}
$$

Note that $u=v u \in C \circ u=C$ (where $v \in C \cap J$ ), so $C$ is an MHP generator.

It is not clear that all of the hypotheses of (2.9)-(2.12) are necessary for the conclusion that $C(=A \circ K \circ u$ or $A K)$ is an MHP generator. In particular, it is possible that the hypotheses of $(2.8)$ are sufficient, but we have been unable to prove this.

In this connection, we recall that, if $A$ is a $\tau$-closed subgroup of $G$, then $C=A \circ u$ is an MHP generator, and the minimal flow it generates is maximally proximal (has no non-trivial proximal extensions). Hence $C=A K$ for some $K \subset J$ and an interesting problem is to determine the subset $K$ of $J$ such that $C=A \circ u=A K$. More generally, if $L \subset J$ is such that $C=A \circ L$ is an MHP generator, for which subsets $K$ of $J$ is it the case that $C=A K(=A \circ K)$ ?

\section{MHP flows and disjointness}

Recall that the minimal flows $X$ and $Y$ are disjoint $(X \perp Y)$ if the product flow $X \times Y$ is minimal. If $X, Y$ and $Z$ are minimal flows, and $\phi: X \rightarrow Z, \psi: Y \rightarrow Z$ are homomorphisms, we say that $X$ and $Y$ are (relatively) disjoint over $Z$ (and write $X \perp_{z} Y$, where the homomorphisms are understood) if the set

$$
R_{\phi \psi}=[(x, y) \mid \phi(x)=\psi(y)]
$$

is a minimal subset of $X \times Y$. In this case, we also say that the homomorphisms $\phi$ and $\psi$ are disjoint (and write $\phi \perp \psi$ ).

Also, if $x_{0} \in X$ and $y_{0} \in Y$, we say that $X$ is relatively disjoint from $Y$ with respect to $x_{0}$ and $y_{0}$ (and write $X \perp_{\left(x_{0}, y_{0}\right)} Y$ ) if there is a minimal flow $Z$ and homomorphisms $\phi: X \rightarrow Z, \psi: Y \rightarrow Z$ with $\phi\left(x_{0}\right)=\psi\left(y_{0}\right)$ such that $\phi \perp \psi$. We now show that, if $X \perp_{\left(x_{0}, y_{0}\right)} Y$, the flow $Z$ in the preceding definition is unique. Precisely, we have:

(3.1) Theorem. Suppose $\phi: Y \rightarrow Z, \quad \psi: Y \rightarrow Z, \quad \phi^{\prime}: X \rightarrow Z^{\prime}, \quad \psi^{\prime}: Y \rightarrow Z^{\prime}$ are homomorphisms of minimal flows with $\phi\left(x_{0}\right)=\psi\left(y_{0}\right), \phi^{\prime}\left(x_{0}\right)=\psi^{\prime}\left(y_{0}\right)$, and $\phi \perp \psi$, $\phi^{\prime} \perp \psi^{\prime}$. Then $Z$ is isomorphic with $Z^{\prime}$. 
Proof. Write $R$ for $R_{\phi \psi}$ and $R^{\prime}$ for $R_{\phi^{\prime} \psi^{\prime}}$. Since $\phi \perp \psi, \phi^{\prime} \perp \psi^{\prime}$, then $R$ and $R^{\prime}$ are minimal, and $\left(x_{0}, y_{0}\right) \in R \cap R^{\prime}$, so $R=R^{\prime}$. Let

$$
\begin{gathered}
z=\phi\left(x_{0}\right)=\psi\left(y_{0}\right), \\
z^{\prime}=\phi^{\prime}\left(x_{0}\right)=\psi^{\prime}\left(y_{0}\right) .
\end{gathered}
$$

If $p, q \in M$ with $z p=z q$, then

$$
\phi\left(x_{0} p\right)=z p=z q=\phi\left(y_{0} q\right)
$$

so

$$
\left(x_{0} p, y_{0} q\right) \in R=R^{\prime},
$$

and

$$
z^{\prime} p=\phi^{\prime}\left(x_{0} p\right)=\psi^{\prime}\left(y_{0} q\right)=z^{\prime} q .
$$

Thus the map $\alpha: Z \rightarrow Z^{\prime}, \alpha(z p)=z^{\prime} p$, is well defined. By symmetry, the map $\alpha^{\prime}: Z^{\prime} \rightarrow Z, \alpha^{\prime}\left(z^{\prime} p\right)=z p$, is well defined and $\alpha$ is an isomorphism.

We adopt the following notational conventions. If $C$ and $D$ are almost periodic points in $2^{M}$, we write $C \rightarrow D$ if there is a homomorphism $\phi$ from $\overline{C T}$ to $\overline{D T}$ with $\phi(C)=D$. We also write $\phi: C \rightarrow D$ to denote this (unique) homomorphism.

If $C, D$, and $E$ are almost periodic points of $2^{M}$, we write $C \perp_{E} D$ if $C \rightarrow E$, $D \rightarrow E$ and the flows $\overline{C T}$ and $\overline{D T}$ are disjoint over $\overline{E T}$. (If $\overline{C T}$ is disjoint from $\overline{D T}$, we write $C \perp D$.)

(3.2) Lemma. Let $C, D$ and $E$ be MHP generators with $C \subset E$ and $D \subset E$. Then the following are equivalent:

(a) $C \perp_{E} D$;

(b) $C \circ p \cap D \circ q \neq \varnothing$, for every $p, q \in M$ with $E \circ p=E \circ q$;

(c) $C \circ D=E$.

Proof. Let $\phi: C \rightarrow E, \psi: D \rightarrow E$. Suppose $C \perp_{E} D$. Let

$$
\mathscr{L}=[(C \circ p, D \circ q) \mid C \circ p \cap D \circ q \neq \varnothing \text { and } E \circ p=E \circ q] .
$$

Then $\mathscr{L}$ is a closed invariant and non-empty (since $(C, D)=(C \circ u, D \circ u) \in \mathscr{L}$ ) subset of $R_{\phi \psi}$. By the assumed relative disjointness,

$$
\mathscr{L}=R_{\phi \psi}
$$

That is,

$$
C \circ p \cap D \circ q \neq \varnothing, \quad \text { whenever } E \circ p=E \circ q \text {. }
$$

Next suppose $(b)$ holds, and let $l \in E$. Then

$$
E \circ l=E=E \circ u,
$$

so

$$
C \circ \ln D=C \circ \ln D \circ u \neq \varnothing,
$$

and there is a $d \in D$ with $d \in C \circ l$. Then

$$
l \in C \circ l=C \circ d \subset C \circ D .
$$

Thus

$$
E \subset C \circ D \subset E \circ E=E
$$


Suppose $(c)$ holds. If $(C \circ p, D \circ q) \in R_{\phi \psi}$, then

$$
E \circ p=E \circ q \text {, }
$$

so

$$
p \in E \circ q=C \circ D \circ q .
$$

Then $p \in C \circ r$ for some $r \in D \circ q$, so

$$
C \circ r=C \circ p, \quad D \circ r=D \circ q
$$

and

$$
(C \circ p, D \circ q)=(C \circ r, D \circ r) .
$$

Therefore $R_{\phi \psi}$ is minimal, and $(a)$ is proved.

(3.3) Corollary. Let $C$ and $D$ be MHP generators, $\mathscr{C}=\overline{C T}$, and $\mathscr{D}=\bar{D} \bar{T}$. Then the following are equivalent:

(1) the flows $\mathscr{C}$ and $\mathscr{D}$ are disjoint;

(2) $C \circ p \cap D \circ q \neq \varnothing$, for all $p, q \in M$;

(3) $C \circ D=M$.

Condition (2) may be paraphrased as 'disjointness of flows is equivalent to nondisjointness of sets'. Note that it implies the known (necessary) condition, in terms of groups, $A B=G[5]$ (which is sufficient in case $T$ is strongly amenable).

In [3], it is shown that disjointness is a 'highly proximal invariant'. That is, disjointness of two flows depends only on their highly proximal equivalence classes. This implies:

(3.4) Corollary. Let $X$ and $Y$ be minimal flows, $x \in X, y \in Y, \gamma: M \rightarrow X, \delta: M \rightarrow Y$, defined by $\gamma(p)=x p, \delta(p)=y p(p \in M)$. Then $X$ and $Y$ are disjoint if and only if

$$
\gamma^{-1}(x) \circ \delta^{-1}(y) \circ u=M \text {. }
$$

Now we consider disjointness (and relative disjointness) of flows defined by MHP generators.

If $C \in 2^{M}$ with $C \circ u=C$, we write $C^{*}$ for the MHP generator

$$
[r \in M \mid C \circ r=C \circ u] \circ u \text {. }
$$

Note that if $\gamma: M \rightarrow \mathscr{X}=\overline{C T}, \gamma(p)=C \circ p$, then

$$
C^{*}=\gamma^{-1}(C) \circ u
$$

so that

$$
\mathscr{X}^{*}=\overline{C^{*} T}
$$

If $C$ contains an idempotent, then $C^{*} \subset C$.

(3.5) THEOREM. Let $C$ and $D$ be MHP generators. Then the following are equivalent:

(a) $C \circ D$ is an MHP generator;

(b) $D \circ C \subset C \circ D$;

(c) $D \circ C=C \circ D$;

(d) $C \rightarrow C \circ D$;

(e) $D \circ C \rightarrow C \circ D$; 
(f) there is an MHP generator $E$ such that $C \perp_{E} D$;

(g) $C \rightarrow(C \circ D)^{*}$;

(h) $C \subset(C \circ D)^{*}$;

(i) $(D \circ C)^{*} \subset(C \circ D)^{*}$.

Proof. $(a) \Rightarrow(b) . D \circ C \subset(C \circ D) \circ(C \circ D)=C \circ D$.

$(a) \Rightarrow(c)$. This follows from (3.2), since the condition $C \perp_{E} D$ is symmetric in $C$ and $D$.

$(a) \Rightarrow(d) . C \subset C \circ D$ and since both $C$ and $C \circ D$ are MHP generators, we have $C \rightarrow C \circ D$.

The implication $(c) \Rightarrow(b)$ is obvious, and $(b) \Rightarrow(a)$ since

$$
(C \circ D) \circ(C \circ D)=C \circ(D \circ C) \circ D \subset C \circ(C \circ D) \circ D=C \circ D \text {. }
$$

$(d) \Rightarrow(a)$. By $(d) C \rightarrow C \circ D$ and always $D \rightarrow C \circ D$. Then

$$
C=C^{*} \rightarrow(C \circ D)^{*} \text { and } D=D^{*} \rightarrow(C \circ D)^{*} \text {, }
$$

so

$$
C \subset(C \circ D)^{*}, \quad D \subset(C \circ D)^{*}
$$

and

$$
C \circ D \subset(C \circ D)^{*} \circ(C \circ D)^{*}=(C \circ D)^{*} .
$$

Since always $(C \circ D)^{*} \subset C \circ D$, we have

$$
C \circ D=(C \circ D)^{*}
$$

which is an MHP generator. Now $(f) \Leftrightarrow(a)$ follows from (3.2) and $(e) \Rightarrow(d)$ since

$$
C \rightarrow D \circ C \rightarrow C \circ D \text {. }
$$

$(g) \Rightarrow(a) . C \rightarrow(C \circ D)^{*}$ is equivalent to $C \subset(C \circ D)^{*}$. Always $D \rightarrow C \circ D$, so

$$
D \rightarrow(C \circ D)^{*}, \quad C \circ D \subset(C \circ D)^{*}
$$

and

$$
\begin{gathered}
C \circ D \subset(C \circ D)^{*} \subset C \circ D \text { and } C \circ D=(C \circ D)^{*} . \\
(i) \Rightarrow(h) . C \rightarrow D \circ C \text {, so } C \rightarrow(D \circ C)^{*} \text {, and } \\
C \subset(D \circ C)^{*} \subset(C \circ D)^{*} \text {. }
\end{gathered}
$$

Since $(c) \Rightarrow(e)$ and $(c) \Rightarrow(i)$ are obvious, as is the equivalence of $(h)$ with $(g)$, the proof is completed.

Note that if the MHP generators $C$ and $D$ satisfy the conditions of the preceding theorem, then $C \perp_{C \circ D} D$ (that is, the MHP generator $E$ in $(f)$ is $C \circ D$ ).

(3.6) LEMMA. Let $\Sigma$ be a non-empty collection of MHP generators and let

$$
C^{\#}=\bigcap[C \mid C \in \Sigma] \text {. }
$$

Then $D=C^{\#} \circ u$ is an MHP generator.

Proof. Since $D \subset C$ for all $C \in \Sigma$,

$$
D \circ D \subset C \circ C=C
$$

so

$$
D \circ D \subset C^{\#}
$$


hence

$$
D \circ D=D \circ D \circ u \subset C^{*} \circ u=D .
$$

(3.7) LeMmA. Let $C, E$ be MHP generators with $C \subset E$. Then there is a minimal (with respect to inclusion) MHP generator $D$ such that $C \circ D=E$.

Proof. Let $\Delta$ be the collection of MHP generators $D^{\prime}$ with $C \circ D^{\prime}=E$. Note that $E \in \Delta$, so $\Delta \neq \varnothing$. Let $\Delta^{\prime}$ be a totally ordered subfamily of $\Delta$,

$$
D_{0}=\bigcap\left[D^{\prime} \mid D^{\prime} \subset \Delta^{\prime}\right]
$$

By (3.6), $D_{0} \circ u$ is an MHP generator. Since $\Delta^{\prime}$ is totally ordered, it follows easily that

$$
C \circ D_{0}=E \text {, }
$$

and therefore

$$
C \circ\left(D_{0} \circ u\right)=\left(C \circ D_{0}\right) \circ u=E \circ u=E .
$$

Thus Zorn's lemma applies to $\Delta$.

We may interpret (3.7) as follows. If $\phi: X \rightarrow Z$ is a homomorphism of MHP minimal flows, then there is an MHP minimal flow $Y$ and a homomorphism $\psi: Y \rightarrow Z$ such that $\psi$ is 'maximally' disjoint from $\phi$. (The precise statement is in theorem 4.13.)

\section{Lifting properties}

In this section we shall consider the commutative diagram of minimal flows and homomorphisms shown as (\#). We shall be concerned with the relation between dynamical properties on the upper and lower levels of the diagram. The main interest is the case that the vertical arrows are proximal or highly proximal, but this is not assumed unless it is explicitly stated.

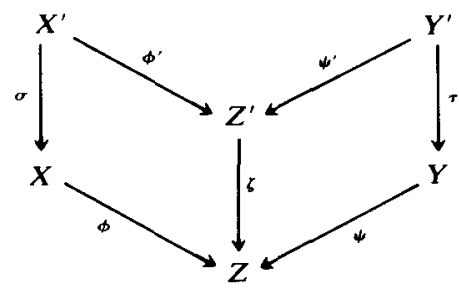

(\#)

We recall the following definitions.

Let $\Lambda$ be an index set of cardinality $>1$ and let $\phi_{\lambda}: X_{\Lambda} \rightarrow Y(\lambda \in \Lambda)$ be homomorphisms of minimal flows (with the same codomain $Y$ ). The collection $\left(\phi_{\lambda}\right)_{\lambda \in \Lambda}$ is said to be Bronstein if the subset $R_{\left(\phi_{\lambda}\right)_{\lambda \in \Lambda}}$ of $\times X_{\lambda}$ defined by

$$
R_{\left(\phi_{\lambda}\right)_{\lambda \in \Lambda}}=\left[\left(x_{\lambda}\right)_{\lambda \in \Lambda} \mid \text { for some } y \in Y, \phi_{\lambda}\left(x_{\lambda}\right)=y \text {, for all } \lambda \in \Lambda\right]
$$

has a dense set of almost periodic points. (This is usually called the generalized Bronstein condition [10].) A homomorphism of minimal flows $\phi: X \rightarrow Y$ will be called Bronstein (' $\phi$ satisfies the Bronstein condition') if $(\phi, \phi)$ is Bronstein; that 
is, the relation

$$
R_{\phi}=R_{\phi \phi}=\left[\left(x, x^{\prime}\right) \in X \times X \mid \phi(x)=\phi\left(x^{\prime}\right)\right]
$$

has a dense set of almost periodic points. Finally, the minimal flow $X$ is Bronstein if the homomorphism $X \rightarrow 1$ is Bronstein. (This is always the case if the acting group $T$ is strongly amenable [7].)

The homomorphism $\phi: X \rightarrow Y$ is called RIC (relatively incontractible) if

$$
x_{0} F^{0} p=\phi^{-1}\left(y_{0} p\right) \quad(p \in M),
$$

where $x_{0} \in X$ with $x_{0} u=x_{0}, y_{0}=\phi\left(x_{0}\right)$, and $F=\left[\alpha \in G / y_{0} \alpha=y_{0}\right]$. The homomorphism $\phi: X \rightarrow Y$ is RIC if and only if $\phi \perp \psi$ for every proximal extension $\psi: Z \rightarrow Y$ ([7], $(X, 1.3)$ ), so the notion of RIC extension is independent of the choice of a basepoint.

If $x \in X$, we write $J(x)$ for the set of $u \in J$ such that $x u=x$.

(4.1) Theorem. (1) The homomorphism $\phi: X \rightarrow Y$ of minimal flows is Bronstein if and only if, for every $x \in X$ and $u \in J(x)$,

$$
R_{\phi}=\overline{\left(\{x\} \times \phi^{-1}(\phi(x) u) T\right.} .
$$

(2) Let $\phi: X \in Y$ and $\psi_{\lambda}: Z_{\lambda} \rightarrow Y(\lambda \in \Lambda)$, be homomorphisms of minimal flows, and suppose that each $\psi_{\lambda}$ is RIC. Then $\left(\phi,\left(\psi_{\lambda}\right)_{\lambda \in \Lambda}\right)$ is Bronstein. (In particular, a RIC extension is Bronstein.)

Proof. (1) Let $\Omega_{\phi}$ denote the almost periodic points of $R_{\phi}$. Let $x \in X, u \in J(x)$, and let

$$
L=\left(\{x\} \times \phi^{-1}(\phi(x)) u\right) T .
$$

Then $L \subset \Omega_{\phi}$ and, if $\bar{L}=R_{\phi}$, then $\phi$ is Bronstein. Suppose that $\phi$ is Bronstein. We first show that $\Omega_{\phi} u \subset \bar{L}$. Let

$$
\left(x^{\prime}, y^{\prime}\right)=\left(x^{\prime}, y^{\prime}\right) u \in \Omega_{\phi}
$$

so

$$
x^{\prime}=x \alpha, \quad y^{\prime}=x \beta
$$

where $\alpha, \beta \in G$. Then

$$
\phi(x \alpha)=\phi(x \beta)
$$

so

$$
\phi\left(x \beta \alpha^{-1}\right)=\phi(x)
$$

and

$$
\left(x, x \beta \alpha^{-1}\right) \in\{x\} \times \phi^{-1}(\phi(x)) u .
$$

Then

$$
\left(x^{\prime}, y^{\prime}\right)=\left(x, x \beta \alpha^{-1}\right) \in \bar{L} .
$$

Finally, if $\left(x^{\prime}, y^{\prime}\right) \in \Omega_{\phi}$, then

$$
\left(x^{\prime}, y^{\prime}\right)=\left(x^{\prime} u, y^{\prime} u\right) w,
$$

for some minimal idempotent $w$, and

$$
\left(x^{\prime}, y^{\prime}\right) \in \overline{\left(\Omega_{\phi} u\right) T} \subset \bar{L} .
$$


This completes the proof of (1). The proof of (2) is straightforward and is therefore omitted.

Before discussing (\#) we consider conditions under which a homomorphism $\phi: C \rightarrow$ $D$ of MHP generators is Bronstein or RIC. These criteria will be applied at the end of the paper.

(4.2) Theorem. Let $C$ and $D$ be MHP generators with $C \subset D$. Let $\phi: C \rightarrow D$. Then

(1) $\phi$ is RIC if and only if $D u \circ C=D$;

(2) $\phi$ is Bronstein if and only if $C \circ D u \circ C=D$.

Proof. (1) $D u \circ u$ is an MHP generator and the minimal flow $\overline{(D u \circ u) T}$ is the maximal proximal extension of $\overline{D T}$. It follows from (4.1) (2) that $\phi: C \rightarrow D$ is $\mathrm{RIC}$ if and only if

$$
D u \circ C=D u \circ u \circ C=D .
$$

(2) Suppose $C \circ D u \circ C=D$. Let $(C \circ p, C \circ q) \in R_{\phi}$, so $D \circ p=D \circ q$. Then

$$
q \in C \circ D u \circ C \circ q=D \circ q=D \circ p=C \circ(D u \circ C \circ p) \text {. }
$$

Then there is an $r \in D u \circ C \circ p$, with $q \in C \circ r$, so

$$
C \circ q=C \circ r \text {. }
$$

Let $r \in D u \circ s$, with $s \in C \circ p$. Let $\left(t_{i}\right)$ and $\left(f_{i}\right)$ be nets with

$$
\left(t_{i}\right) \subset T, \quad t_{i} \rightarrow s, \quad\left(f_{i}\right) \subset D u \quad \text { and } \quad f_{i} t_{i} \rightarrow r .
$$

Then

$$
C \circ q=\lim C \circ f_{i} t_{i} \text { and } C \circ p=C \circ s=\lim C t_{i}
$$

so

$$
(C \circ p, C \circ q)=\lim \left(C, C \circ f_{i}\right) t_{i} \in \overline{(\{C\} \times\{C \circ f \mid f \in D u\}) T} .
$$

By (4.1) (1), $\phi$ is Bronstein.

Conversely, suppose that $\phi$ is Bronstein and let $d \in D$. Then $(C, C \circ d) \in R_{\phi}$, so there are nets $\left(t_{i}\right) \subset T,\left(f_{i}\right) \subset D u$ with

$$
(C, C \circ d)=\lim \left(C, C \circ f_{i}\right) t_{i}
$$

(4.1 (1) again). Let

$$
c=\lim u t_{i} \in \lim C t_{i}=C
$$

and let

$$
q=\lim f_{i} t_{i} \in D u^{\circ} \mathrm{c}
$$

Then

$$
C \circ d=C \circ q
$$

so

$$
d \in C \circ d=C \circ q \subset C \circ D u \circ C
$$

Hence

$$
D \subset C \circ D u \circ C \subset D \circ D \circ D=D
$$

and

$$
C \circ D u \circ C=D
$$


Now we turn to a detailed discussion of (\#).

(4.3) LemMA. In (\#), suppose $(\phi, \psi)$ is Bronstein and $\zeta$ is proximal. Then

$$
(\sigma \times \tau)\left(\boldsymbol{R}_{\phi^{\prime} \psi^{\prime}}\right)=\boldsymbol{R}_{\phi \psi}
$$

Proof. Let $(x, y)$ be an almost periodic point in $R_{\phi \psi}$ and let $\left(x^{\prime}, y^{\prime}\right)$ be almost periodic in $X^{\prime} \times Y^{\prime}$ with

$$
\sigma\left(x^{\prime}\right)=x, \quad \tau\left(y^{\prime}\right)=y .
$$

Then, by the commutativity of (\#),

$$
\zeta \phi^{\prime}\left(x^{\prime}\right)=\zeta \psi^{\prime}\left(y^{\prime}\right)
$$

so

$$
\left(\phi^{\prime}\left(x^{\prime}\right), \psi^{\prime}\left(y^{\prime}\right)\right) \in P,
$$

and since also $\left(\phi^{\prime}\left(x^{\prime}\right), \psi^{\prime}\left(y^{\prime}\right)\right)$ is almost periodic,

$$
\phi^{\prime}\left(x^{\prime}\right)=\psi^{\prime}\left(y^{\prime}\right) \text { and }\left(x^{\prime}, y^{\prime}\right) \in R_{\phi^{\prime} \psi^{\prime}} .
$$

Since almost periodic points are dense in $R_{\phi \psi}$ we have

$$
(\sigma \times \tau)\left(R_{\phi^{\prime} \psi^{\prime}}\right) \supset R_{\phi \psi} .
$$

The opposite inclusion always holds, so the proof is completed.

(4.4) LEMMA. Let $\phi: X \rightarrow Y, \psi: Y \rightarrow Z$ be homomorphisms of minimal flows with $\phi$ open and $\psi$ HP. Let $z \in Z, y \in \psi^{-1}(z)$, and $p \in M$. Then

$$
\phi^{-1}(y p)=\phi^{-1}\left(\psi^{-1}(z)\right) \circ p .
$$

Proof. Since $\phi$ is open, the map $A \mapsto \phi^{-1}(A)$ of $2^{Y}$ to $2^{X}$ is continuous. Then

$$
\phi^{-1}\left(\psi^{-1}(z)\right) \circ p=\phi^{-1}\left(\psi^{-1}(z) \circ p\right)=\phi^{-1}(y p) .
$$

(4.5) LEMMA. In (\#), suppose that $\phi, \phi^{\prime}$ and $\psi^{\prime}$ are open and that $\zeta$ is HP. Then

$$
(\sigma \times \tau)\left(R_{\phi^{\prime} \psi^{\prime}}\right)=R_{\phi \psi}
$$

Proof. Let $z_{0} \in Z$ and $z_{1} \in \zeta^{-1}\left(z_{0}\right)$. Note that

$$
R_{\phi^{\prime} \psi^{\prime}}=\bigcup\left[\phi^{\prime-1}\left(z_{1} p\right) \times \psi^{\prime-1}\left(z_{1} p\right) \mid p \in M\right] .
$$

By (4.4),

$$
R_{\phi^{\prime} \psi^{\prime}}=\left[\left(\phi^{\prime-1}\left(\zeta^{-1}\left(z_{0}\right) \circ p\right)\right) \times\left(\psi^{\prime-1}\left(\zeta^{-1}\left(z_{0}\right) \circ p\right)\right) \mid p \in M\right]
$$

Now

$$
\sigma\left(\phi^{\prime-1}\left(\zeta^{-1}\left(z_{0}\right) \circ p\right)\right)=\phi^{-1}\left(z_{0}\right) \circ p=\phi^{-1}\left(z_{0} p\right)
$$

(since $\phi$ is open), and

$$
\tau\left(\psi^{\prime-1}\left(\zeta^{-1}\left(z_{0}\right)\right) \circ p\right)=\psi^{-1}\left(z_{0}\right) \circ p
$$

Also

$$
\psi^{-1}\left(z_{0} p\right)=\bigcup\left[\psi^{-1}\left(z_{0}\right) \circ q \mid z_{0} q=z_{0} p\right]
$$

so

$$
\begin{aligned}
(\sigma \times \tau)\left(R_{\phi^{\prime} \psi^{\prime}}\right) & =\bigcup\left[\phi^{-1}\left(z_{0} p\right) \times \psi^{-1}\left(z_{0}\right) \circ p \mid p \in M\right] \\
& =\bigcup\left[\phi^{-1}\left(z_{0} p\right) \times \psi^{-1}\left(z_{0} p\right) \mid p \in M\right]=R_{\phi \psi}
\end{aligned}
$$


(4.6) LeMMA. Let $\phi: X \rightarrow Z$ and $\psi: Y \rightarrow Z$ be homomorphisms of minimal flows. Suppose that $(\phi, \psi)$ is Bronstein or that one of $\phi$ and $\psi$ is open. Let $W$ be a non-empty open set in $R_{\phi \psi}$. Then there are open subsets $U$ of $X$ and $V$ of $Y$ such that:

(i) $(U \times V) \cap R_{\phi \psi}$ is a non-empty subset of $W$;

(ii) if $x \in U$, there is a $y \in V$ with $(x, y) \in R_{\phi \psi}$.

Proof. Define $\theta: R_{\phi \psi} \rightarrow Z$ by

$$
\theta=\left.(\phi \times \psi)\right|_{R_{\phi \psi}},
$$

and choose $U_{0}$ and $V_{0}$ open in $X$ and $Y$ respectively with $\varnothing \neq U_{0} \times V_{0} \cap R_{\phi \psi} \subset W$. It is sufficient to prove that

$$
W^{\prime}=\operatorname{int} \theta\left(\left(U_{0} \times V_{0}\right) \cap R_{\phi \psi}\right)=\operatorname{int}\left(\phi\left(U_{0}\right) \cap \psi\left(V_{0}\right)\right)
$$

is non-empty. (For, in this case,

$$
U=U_{0} \cap \phi^{-1}\left(W^{\prime}\right) \text { and } V=V_{0} \cap \psi^{-1}\left(W^{\prime}\right)
$$

satisfy (i) and (ii).)

If $(\phi, \psi)$ is Bronstein, let $\left(x_{0}, y_{0}\right)$ be an almost periodic point of $U_{0} \times V_{0} \cap R_{\phi \psi}$, and define

$$
\tilde{W}=\left(U_{0} \times V_{0}\right) \cap R_{\phi \psi} \cap \overline{\left(x_{0}, y_{0}\right) T}
$$

Since $\overline{\left(x_{0}, y_{0}\right) T}$ is minimal,

$$
\text { int } \theta\left(\left(U_{0} \times V_{0}\right) \cap R_{\phi \psi}\right) \supset \text { int } \theta(\tilde{W}) \neq \varnothing .
$$

If $\phi$ is open, then

$$
\tilde{V}=V_{0} \cap \psi^{-1} \phi\left(U_{0}\right)
$$

is open and non-empty, so

$$
\text { int }\left(\phi\left(U_{0}\right) \cap \psi\left(V_{0}\right)\right) \supset \text { int } \psi(\tilde{V}) \neq \varnothing .
$$

(If $\psi$ is open, the argument is completely analogous.)

(4.7) Corollary. Let $\phi: X \rightarrow Z$ and $\psi: Y \rightarrow Z$ be homomorphisms of minimal flows, and suppose that one of $\phi$ and $\psi$ is open. If $\phi$ is point distal, then $(\phi, \psi)$ is Bronstein.

Proof. Let $W$ be a open subset of $R_{\phi \psi}$ and let $U \subset X$ and $V \subset Y$ be as in (4.6). Let $x \in U$ be such that, if $\phi(x)=\phi\left(x^{\prime}\right)$, then $x$ and $x^{\prime}$ are distal. Let $y \in V$ be such that $\phi(x)=\psi(y)$, and let $w$ be a minimal idempotent such that $y w=y$. Then

$$
\phi(x w)=\psi(y w)=\psi(y)=\phi(x)
$$

and $x$ is proximal to $x w$, so $x w=x$. Then $(x, y) \in U \times V \subset W$ is an almost periodic point.

(4.8) Corollary. An open point distal homomorphism of minimal flows is RIC. Proof. Let $\phi: X \rightarrow Y$ be open point distal, and let $\psi: Z \rightarrow Y$ be proximal. Now, by the previous corollary, $R_{\phi \psi}$ has a dense set of almost periodic points. But, since $\psi$ is proximal, the projection $R_{\phi \psi} \rightarrow X$ is proximal, and so $R_{\phi \psi}$ is minimal. Thus $\phi \perp \psi$, and by (4.1) (2), $\phi$ is RIC. 
(4.9) Theorem. In (\#), suppose that $\sigma$ and $\tau$ are HP. If $\left(\phi^{\prime}, \psi^{\prime}\right)$ is Bronstein or $\phi^{\prime}$ is open or $\psi^{\prime}$ is open, then

$$
\sigma \times \tau: R_{\phi^{\prime} \psi^{\prime}} \rightarrow(\sigma \times \tau)\left(R_{\phi^{\prime} \psi^{\prime}}\right)
$$

is irreducible.

Proof. Let $W^{\prime}$ be an open subset of $R_{\phi^{\prime} \psi^{\prime}}$. Let $U^{\prime} \subset X^{\prime}$ and $V^{\prime} \subset Y^{\prime}$ be open sets as in (4.6). Then

$$
U^{2}=\sigma^{-1}\left(X-\sigma\left(X^{\prime}-U^{\prime}\right)\right)
$$

is open and non-empty (since $\sigma$ is irreducible) and int $\phi^{\prime}\left(U^{2}\right)$ is a non-empty subset of $\psi^{\prime}\left(V^{\prime}\right)$. Hence

$$
V^{2}=\psi^{\prime-1}\left(\text { int } \phi^{\prime}\left(U^{2}\right)\right) \cap V^{\prime}
$$

is non-empty and open. Define

$$
V^{3}=\tau^{-1}\left(Y-\left(Y^{\prime}-V^{2}\right)\right) .
$$

Then $V^{3} \subset V^{2}$. Since $\tau$ is irreducible, $V^{3}$ is non-empty and

$$
\psi^{\prime}\left(V^{3}\right) \subset \phi^{\prime}\left(V^{2}\right) \text {. }
$$

Clearly,

$$
U^{2} \times V^{3}=(\sigma \times \tau)^{-1}\left((\sigma \times \tau)\left(U^{2} \times V^{3}\right)\right)
$$

and

$$
\varnothing \neq\left(U^{2} \times V^{3}\right) \cap R_{\phi^{\prime} \psi^{\prime}} \subset W^{\prime} .
$$

Hence $W^{\prime}$ contains a $\sigma \times \tau$ fibre, so $\sigma \times \tau$ is irreducible.

Recall that a flow is (topologically) ergodic if every invariant non-empty open set is dense. In the following lemma, the flows $X$ and $Y$ need not be minimal. We omit the straightforward proof.

(4.10) LeMMA. Let $\phi: X \rightarrow Y$ be an irreducible homomorphism. Then $Y$ is minimal (ergodic) (has a dense set of almost periodic points) if and only if $X$ has the corresponding property.

Given homomorphisms of minimal flows $\phi: X \rightarrow Z, \psi: Y \rightarrow Z$ we apply the results obtained in this section to the diagram ${ }^{*}(\phi, \psi)$ (or just $\left.(*)\right)$. Here (as in $\S 2$ ), $X^{*}$, $Y^{*}$ and $Z^{*}$ are the MHP extensions of $X, Y$ and $Z$, respectively, $\sigma, \zeta$ and $\tau$ are the natural HP homomorphisms, and $\phi^{*}$ and $\psi^{*}$ are the induced (open) maps.

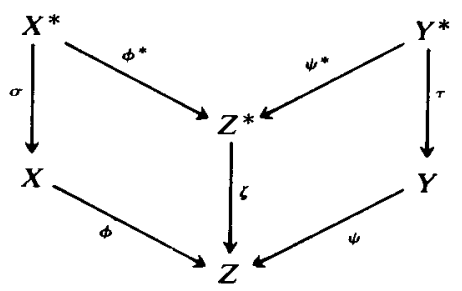

$(*)$ 
(4.11) Theorem. Consider $(*)$.

(1) If $(\phi, \psi)$ is Bronstein, then $\left(\phi^{*}, \psi^{*}\right)$ is Bronstein.

(2) If $\phi \perp \psi$ then $\phi^{*} \perp \psi^{*}$.

(3) Suppose $(\phi, \psi)$ is Bronstein or $\phi($ or $\psi)$ is open. Then

(a) $\phi \perp \psi$ if and only if $\phi^{*} \perp \psi^{*}$;

(b) $R_{\phi \psi}$ is ergodic if and only if $\boldsymbol{R}_{\phi^{*} \psi^{*}}$ is ergodic.

(4) Suppose $\phi$ or $\psi$ is open. Then $(\phi, \psi)$ is Bronstein if and only if $\left(\phi^{*}, \psi^{*}\right)$ is Bronstein.

Proof. Use (4.3) (or 4.5), (4.9) and (4.10).

(4.12) THEOREM. Let $\phi: X \rightarrow Y$ be a homomorphism of minimal flows.

(1) If $\phi$ is Bronstein or RIC, then $\phi^{*}$ has the corresponding property.

(2) If $\phi$ is open, then $\phi$ is Bronstein or RIC if and only if $\phi^{*}$ has the corresponding property.

(3) If $\phi$ is open or Bronstein, then $\phi$ is weakly mixing $\left(R_{\phi \phi}\right.$ is ergodic), if and only if $\phi^{*}$ is weakly mixing.

Proof. Most of the assertions follow directly from (4.11). For the RIC versions of (1) and (2), use the characterization of RIC extensions given in the discussion preceding (4.1).

Now, as promised we generalize (3.7).

(4.13) THEOREM. Let $\phi: X \rightarrow Z$ be an open homomorphism of minimal flows. Then there is a minimal flow $Y$ and a homomorphism $\psi: Y \rightarrow Z$ which is 'maximally disjoint' from $\phi$. That is, $\phi \perp \psi$ and if $\theta: Y^{\prime} \rightarrow Y$ is a homomorphism (with $Y^{\prime}$ minimal) such that $\psi \circ \theta \perp \phi$, then $\theta$ is an isomorphism. Moreover, the flow $Y$ is MHP.

Proof. We first assume that both $X$ and $Z$ are MHP. In this case we may suppose that

$$
X=\overline{C T}, \quad Z=\overline{E T},
$$

where $C$ and $E$ are MHP generators with $C \subset E$. Let $D$ be an MHP generator as in (3.7) (that is, $C \circ D=E$, and $D$ is inclusion minimal), let $Y=\overline{D T}$, and $\psi: Y \rightarrow Z$ be defined by

$$
\psi(D \circ p)=E \circ p .
$$

Now, suppose $\theta: Y^{\prime} \rightarrow Y$ is a homomorphism of minimal flows with $\psi \circ \theta \perp \phi$. Let $y^{\prime} \in Y^{\prime}$ with

$$
y^{\prime} u=y^{\prime}, \quad \theta\left(y^{\prime}\right)=D,
$$

and $\delta^{\prime}: M \rightarrow Y^{\prime}$

$$
D^{*}=\delta^{\prime-1}(y) \circ u
$$

so

$$
Y^{* *}=\overline{D^{*} T}
$$

$\delta^{*}: Y^{*} \rightarrow Y^{\prime}$, given by

$$
\delta^{*}\left(D^{*} \circ p\right)=y^{\prime} p
$$

is highly proximal. Now $D^{*}$ is an MHP generator with $D^{*}:=D$, and [3, lemma III.1] 
$\psi \theta \delta^{*} \perp \phi$, so

$$
C \circ D^{*}=E \text {. }
$$

By the defining property of $D$, we have $D^{*}=D$, and it follows that $\theta$ is an isomorphism. Thus the theorem is proved if $X$ and $Z$ are MHP.

Now we suppose that $X$ is arbitrary and that $\phi$ is open. Let $\phi^{*}: X^{*} \rightarrow Z^{*}$ be the induced homomorphism of MHP flows. By the first part of the proof there is an MHP minimal flow $Y=Y^{*}$ and a homomorphism $\psi: Y \rightarrow Z^{*}$ such that $\psi$ is maximally disjoint from $\phi^{*}$. If, as in (*), $\zeta: Z^{*} \rightarrow Z$ and $\tilde{\psi}=\zeta \circ \psi: Y \rightarrow Z$, then by (4.11) (3), $\phi \perp \tilde{\psi}$. We show that $\tilde{\psi}$ is maximally disjoint from $\phi$. For suppose $W$ is a minimal flow and $\theta: W \rightarrow Y$ is a homomorphism for which $\phi \perp \tilde{\psi} \circ \theta$. Then, lifting the homomorphisms $\phi: X \rightarrow Z$ and $\psi \circ \theta: W \rightarrow Z$ to their MHP extensions, we have, by (4.11) (2), that

But

$$
\phi^{*} \perp(\tilde{\psi} \circ \theta)^{*} .
$$

$$
(\tilde{\psi} \circ \theta)^{*}=\psi \circ \theta^{*},
$$

so

$$
\phi^{*} \perp \psi \circ \theta^{*} \text {. }
$$

By the maximality of $\psi, \theta^{*}$ is an isomorphism and (recalling that $Y^{*}=Y$ ) it fo!lows that $\theta$ is an isomorphism. The proof is completed.

Note that (4.13) applies if $\phi$ is RIC or if $Z$ is MHP.

In what follows, we write $X \times_{W} Y$ instead of $R_{\phi \psi}$ (where $\phi: X \rightarrow W$ and $\psi: Y \rightarrow W$ are homomorphisms) and write

$$
(\phi, \psi): X \times_{W} Y \rightarrow W
$$

for the homomorphism

$$
(\phi, \psi)(x, y)=\phi(x)=\psi(y) .
$$

Suppose $\phi: X \rightarrow W$ is an open homomorphism of minimal flows and let $\psi: Y \rightarrow W$ be a homomorphism which is maximally disjoint from $\phi$. Let $Z=X \times{ }_{W} Y$, and suppose $\lambda^{\prime}: Z^{\prime} \rightarrow W$ is a homomorphism with $\lambda^{\prime} \perp(\phi, \psi)$. Then

$$
X \times_{W}\left(Y \times_{W} Z^{\prime}\right) \cong\left(X \times_{W} Y\right) \times_{W} Z^{\prime}=Z \times_{W} Z^{\prime}
$$

is minimal, and therefore $Y \times_{W} Z^{\prime}$ is minimal and disjoint from $X$ over $W$. By the assumed maximality of $\psi, Y \times_{W} Z^{\prime}$ is isomorphic with $Y$. But in this case

$$
\left(Y \times_{W} Z^{\prime}\right) \times_{W} Z^{\prime} \cong Y \times_{W} Z^{\prime},
$$

and so $\left(Y \times_{W} Z^{\prime}\right) \times_{W} Z^{\prime}$ is minimal. It follows that $Z^{\prime} \times_{W} Z^{\prime}$ is minimal. But this can only occur if $\lambda^{\prime}: Z^{\prime} \rightarrow W$ is an isomorphism. This proves:

(4.14) THEOREM. If $\phi: X \rightarrow W$ and $\psi: Y \rightarrow W$ are homomorphisms of minimal flows such that $\psi$ is maximally disjoint from $\phi$, then any homomorphism $\lambda^{\prime}: Z^{\prime} \rightarrow W$ which is disjoint from $(\phi, \psi)$ is an isomorphism.

(4.15) Corollary. If $X$ is any minimal flow and $Y$ is a maximal minimal flow such that $X \perp Y$, then

$$
(X \times Y)^{\perp}=\{1\} .
$$


In addition to $(*)$, there are other diagrams derived from (\#) which are naturally associated with a pair of homomorphisms $\phi: X \rightarrow Z, \psi: Y \rightarrow Z$. For instance, the flows on the upper level of (\#) can be the maximal proximal extensions of $X, Y$, and $Z$. Another case of interest is obtained by putting, for $z \in Z$,

and

$$
\begin{aligned}
X^{\prime} & =\left[\phi^{-1}(z) \circ p \mid p \in M\right], \\
Y^{\prime} & =\left[\psi^{-1}(z) \circ p \mid p \in M\right],
\end{aligned}
$$

$$
Z^{\prime}=Z \text {. }
$$

We hope to study these and related constructions in a subsequent paper.

We conclude by obtaining a sufficient condition for a homomorphism of minimal flows to be RIC. A homomorphism $\phi: X \rightarrow Y$ is called regular if, whenever $\left(x_{1}, x_{2}\right) \in$ $R_{\phi}$ is an almost periodic point, there is an automorphism $\theta$ of $X$ such that

$$
\theta\left(x_{1}\right)=x_{2} \text {. }
$$

(So, if $Y=1, \phi: X \rightarrow 1$ is regular if and only if the minimai flow $X$ is regular.)

(4.16) Theorem. Let $C$ and $D$ be MHP generators with $C \subset D$, and let $\phi: C \rightarrow D$.

(1) If $\phi$ is regular, then $D u \circ C$ is an MHP generator.

(2) If $\phi$ is regular and Bronstein, then $\phi$ is $\mathrm{RIC}$.

Proof. (1) If $f \in D u$, then $(C, C \circ f) \in R_{\phi}$ is an almost periodic point, and there is an automorphism $\theta$ of $\overline{C T}$ such that $\theta(C)=C \circ f$, so

$$
f^{-1} \circ \theta: C \rightarrow f^{-1} C \circ f
$$

is an isomorphism. Since $f^{-1} C \circ f$ is an MHP generator (2.5 (4)),

Then, if $f \in D u$, we have

$$
f^{-1} C \circ f=C \text {. }
$$

$$
D u \circ C=D u \circ f^{-1} \circ C \circ f=D u \circ u \circ C \circ f=D u \circ C \circ f,
$$

so

$$
D u \circ C=D u \circ C \circ D u
$$

Hence

$$
D u \circ C \circ D u \circ C=D u \circ C \circ C=D u \circ C .
$$

(2) We have $C \subset D u \circ C$ and, since $D u \circ C$ is an MHP generator,

$$
C \circ D u \circ C=D u \circ C \text {. }
$$

By (4.2) (2),

$$
C \circ D u \circ C=D
$$

so

$$
D u \circ C=D
$$

and, by (4.2) (1), $\phi$ is RIC.

We omit the easy proof of the following lemma.

(4.17) LeMmA. Let $\phi: X \rightarrow Y$ be a regular homomorphism of minimal flows. Then the induced homomorphism $\phi^{*}: X^{*} \rightarrow Y^{*}$ of the MHP extensions is also regular. 
(4.18) THEOREM. Let $\phi: X \rightarrow Y$ be a homomorphism of minimal flows which is open, regular, and Bronstein. Then $\phi$ is RIC.

Proof. The homomorphism $\phi^{*}$ is Bronstein (4.12 (2)) regular (4.17) and open (always). By (4.16) $\phi^{*}$ is RIC. It follows from (4.12) (2) that $\phi$ is RIC.

\section{REFERENCES}

[1] T. E. Armstrong. Gleason spaces and topological dynamics. Indiana Math. J. 27 (1978), 283-292.

[2] J. Auslander. Regular minimals sets, I. Trans Amer. Math. Soc. 123 (1966), 469-479.

[3] J. Auslander \& S. Glasner. Distal and highly proximal extensions of minimal flows. Indiana Math. J. $26(1900), 731-749$.

[4] W. W. Comfort. Ultrafilters: some old and new results. Bull. Amer. Math. Soc. 83 (1977), 417-455.

[5] R. Ellis. Lectures on Topological Dynamics. W. A. Benjamin: New York, 1969.

[6] R. Ellis. The Furstenberg structure theorem. Pacific J. Math. 76 (1978), 345-349.

[7] S. Glasner. Proximal Flows. Lecture Notes in Math. 517. Springer-Verlag: Berlin, 1976.

[8] D. C. McMahon \& T. S. Wu. On the connectedness of homomorphisms in topological dynamics. Trans. Amer. Math. Soc. 217 (1976), 257-270.

[9] W. Parry \& P. Walters. Minimal skew product homeomorphisms and coalescence. Compositio Math. 22 (1970), 283-288.

[10] W. A. Veech. Topological dynamics. Bull. Amer. Math. Soc. 83 (1977), 775-830.

[11] S. Willard. General Topology. Addison Wesley: Reading, Mass., 1970. 